\title{
Pentacam: Principle and Clinical Applications
}

\author{
Rajeev Jain, SPS Grewal
}

Grewal Eye Institute, Chandigarh, India

Rotating Scheimpflug imaging technology is used by instruments such as the Pentacam (Oculus) to measure the anterior and posterior corneal surfaces, as well as other anterior segment structures. Initially, Scheimpflug photography was used to obtain images of lens opacities for objective evaluation, but Pentacam-Scheimpflug camera is much wider in its clinical applications as a diagnostic tool for not only corneal diseases like keratoconus but cataract and refractive surgeons as well as glaucomatologists than the Scheimpflug photography alone. Its applications are growing everyday and its utility is till date unsurpassable in a busy anterior segment ophthalmic practice.

\section{THE SCHEIMPFLUG PRINCIPLE}

The most precise and versatile method to document light scattering and biometry of the anterior eye segment is slit image photography according to Scheimpflug's principle. The Scheimpflug principle, first introduced by Theodor Scheimpflug, ${ }^{1}$ a cartographer of the Austrian navy, describes an optical imaging condition, which allows documentation of an obliquely tilted object with the maximally possible depth of focus and minimal image distortion under given conditions. It is is a geometric rule that describes the orientation of the plane of focus of an optical system (such as a camera) when the lens plane is not parallel to the image plane. Normally, the lens and image (film or sensor) planes of a camera are parallel and the plane of focus (PoF) is parallel to the lens and image planes (Fig. 1A). If a planar subject (such as the side of a building) is also parallel to the image plane, it can coincide with the PoF, and the entire subject can be rendered sharply. If the subject plane is not parallel to the image plane, it will be in focus only along a line where it intersects the PoF, as illustrated in Figure 1B.

When an oblique tangent is extended from the image plane, and another is extended from the lens plane, they meet at a line through which the PoF also passes, as illustrated in Figure 1C (referred as Scheimpflug line). With this condition, a planar subject that is not parallel to the image plane can be completely in focus. The Scheimpflug principle has been applied in ophthalmology to obtain optical sections of the entire anterior segment of the eye, from the anterior surface of the cornea to the posterior surface of the lens.

\section{HISTORY}

Theodor Scheimpflug, born in Vienna on 7 October 1865, as a young and talented naval officer was annoyed by the inaccuracy
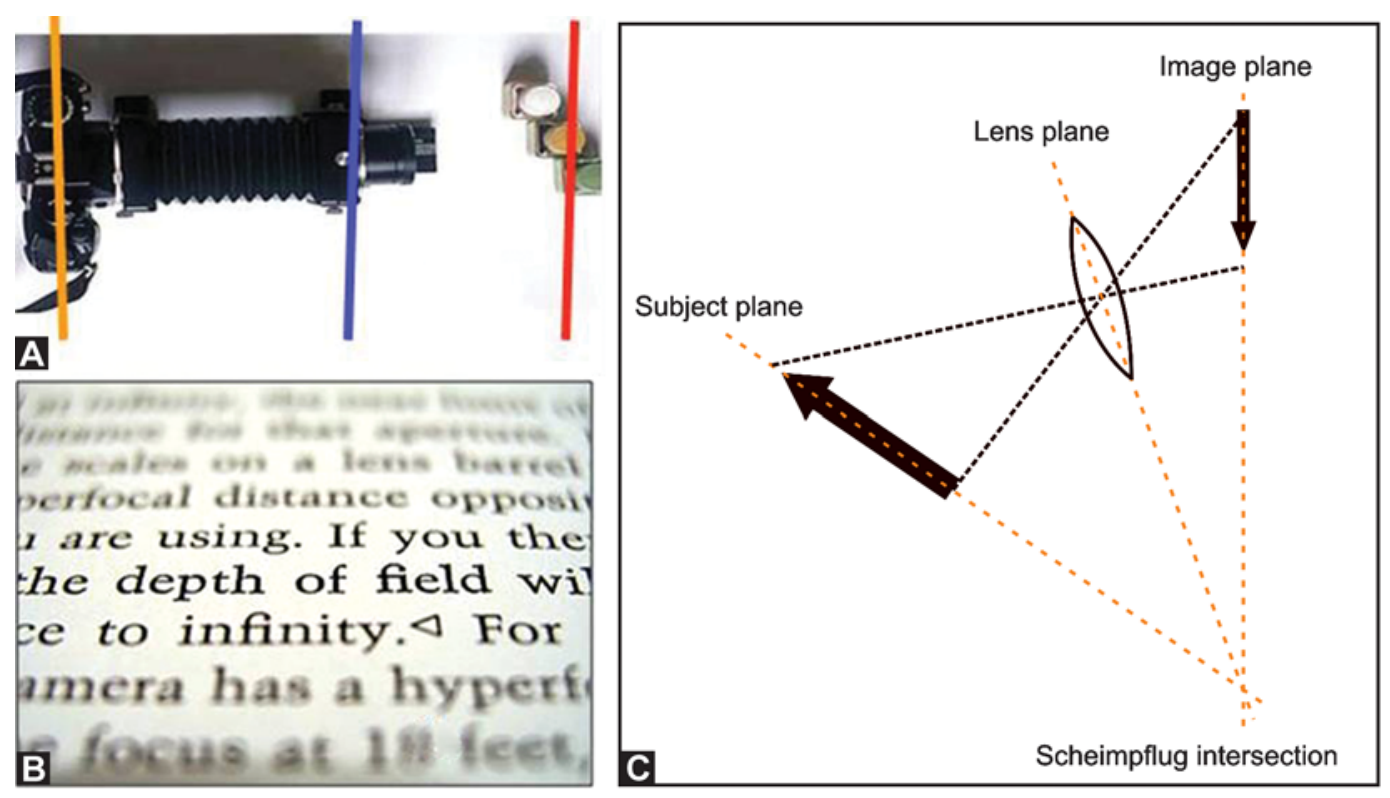

Figs $1 \mathrm{~A}$ to $\mathrm{C}:(\mathrm{A})$ The parallel lens and image (film or sensor) planes of routine camera, (B) The subject plane is not parallel to the image plane, hence it is in focus only along a line where it intersects the point of focus (PoF), (C) In Scheimpflug camera, the subject plane, lens plane and film plane intersect at point of focus (PoF), referred as Scheimpflug line 
of most naval charts, he had to use during his training on various cruises with battleships to become a captain. Because of his scientic interest in geometry, Scheimpflug was granted a sabbatical from the navy and started to study under Professor Mayer in Vienna, where he got in contact with photogrammetry. ${ }^{2}$ He pursued two main scientic and practical goals: the construction of an apparatus for aerial photography and photogrammetry to produce exact undistorted photo maps from landscapes for military aerial reconnaissance, if possible even with contour readings. His second goal was more market oriented: he wanted to use his flying camera for the production of exact photo maps from colonial territories for the economic prot of Austrian foreign trade. ${ }^{3}$ This Scheimpflug principle was introduced into ophthalmology by Drews, Niesel, Brown. ${ }^{4-8}$

The first rotating Scheimpflug camera was designed by Dragomirescu and Hockwin, ${ }^{9-10}$ using black-and-white film for recording.

\section{SCHEIMPFLUG INSTRUMENTATION}

The first Scheimpflug camera for ophthalmic use was Topcon SL-45 Scheimpflug camera. A nonrotating Scheimpflug camera was first produced by the Oxford group, later on marketed as Oxford CASE 2000 (Cataract Assessment Scheimpflug Equipment). ${ }^{11-12}$ The Zeiss SLC was the first rotating video Scheimpflug system. The EAS 1000 (Eye analysis system) was the first electronic rotating Scheimpflug camera, which recorded a retroillumination image as well. ${ }^{13}$

The latest development among ophthalmic camera systems based on Scheimpflug's principle is the Pentacam (Oculus, Wetzlar, Germany) (Fig. 2A), the first multipurpose instrument providing five different measurement options for the anterior eye segment. ${ }^{14-15}$ These are Pachymetry, corneal topography, anterior and posterior corneal curvature, astigmatism determination and Scheimpflug photography of the lens.

The GALILEITM Dual Scheimpflug Analyzer (Zeimer Ophthalmic systems) is another high precision optical system for corneal topography and three dimensional analysis of the anterior eye segment, also based on a Revolving Dual Channel Scheimpflug Camera and a Placido Disk. The principle advantage of Dual Scheimpflug imaging is that corresponding corneal thickness data from each view can simply be averaged to compensate for unintentional misalignment, which results in a corrected measurement value at the corresponding location.

Pentacam- Scheimpflug imaging device is the latest addition with multitask utility and is fast gaining its popularity in usage in ophthalmology clinics.

\section{PENTACAM: BASIC CONSIDERATIONS}

Cross-sectional imaging technology is seldom used for the diagnosis of corneal abnormalities. Computerized tomography and magnetic resonance imaging are time consuming and the resolution of the images is insufficient to study corneal diseases. Ultrasonic biomicroscopy and laser confocal microscopy have a higher resolution and are used as diagnostic tools for the anterior segment of the eye providing images of higher resolution that delineate corneal cellular structures in vivo; however, ultrasonic biomicrosocopy requires an immersion technique and laser confocal microscopy requires direct contact of the transducer to the cornea limiting its usefulness for patient with corneal epithelial problems, infections disorders and postoperative patients. Noncontact methods would be advantageous for corneal conditions associated with epithelial disorders, almost as a rule.

The Pentacam obtains images of the anterior segment by a rotating Scheimpflug camera measurement (Fig. 2B). The camera is a digital CCD camera with synchronous pixel sampling. The light source consists of UV-free blue light emitting diode (LED's) (Fig. 2C) with a wavelength of $475 \mathrm{~nm}$. The system integrates two cameras. One is located in the center for the purposes of detection of the size and orientation of the pupil and to control fixation. The second is mounted on rotating wheel to capture images from the anterior segment. It's a complete picture from anterior surface of the cornea to the posterior surface of the lens. This rotating process supplies pictures in three dimensions and also allows the center of the cornea to be measured precisely. The slit images are photographed on an angle form 0 to 180 degrees to avoid shadows from nose. Every picture is a complete image through the cornea at a specific angle, combination of such slit images creates a real 360 degrees image of the anterior segment. The software utilizes a ray tracing algorithm to construct and calculate the anterior segment. It acquires a total of 50 images in approximately two seconds, extracting 2,760 true elevation points from these images which in turn generates 138,000 true elevation points for both the corneal front and back surfaces, from limbus to limbus, including the center of the cornea, a major advantage over keratometers and Placido-based corneal topographers. The measurement process lasts less than two seconds and minute eye movements are captured and corrected simultaneously.

Scheimpflug photography provides images of the anterior eye segment with minimal distortion. However, the distortion of the camera optics and of the cornea and lens itself distort the image. Therefore, biometrical measurements in the anterior eye segment, such as corneal curvature, changes of lens curvature during accommodation, depth of the anterior chamber and anterior chamber angle, always have to be corrected by specific algorithms. ${ }^{15,16}$ The amount of correction to be performed depends on the depth of the layer in question, meaning that each refractive zone adds a small amount of distortion to the path of the light rays. The Pentacam is the only camera whose software includes an algorithm for automatic distortion correction. Correction of image distortion plays an important role in all Scheimpflug application to corneal biometry, refractive 


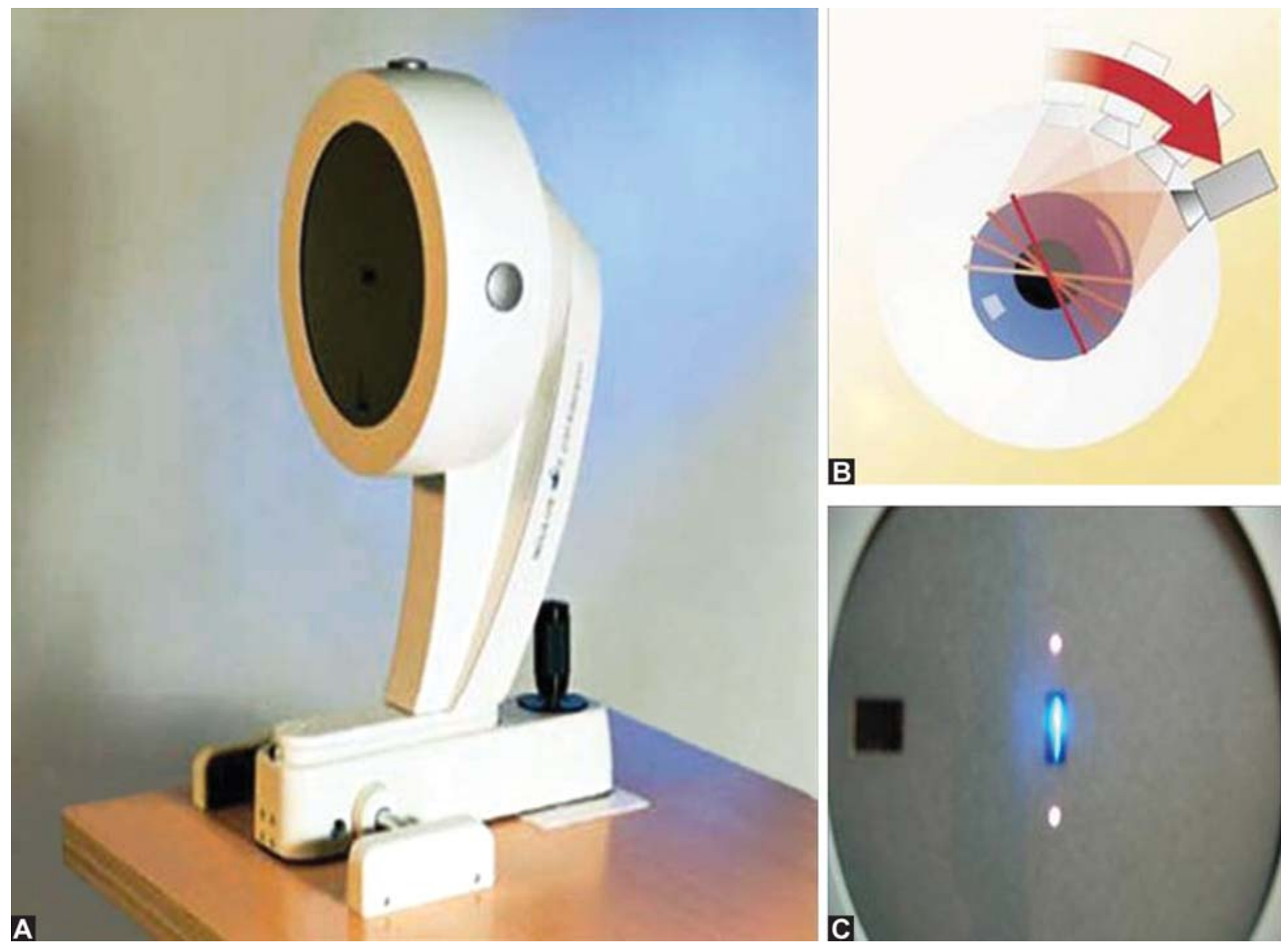

Figs 2A to C: $(A)$ Pentacam, $(B)$ Diagrammatic representation of rotating camera (C) The light source consists of UV-free blue light emitting diode (LED's)—Patient's view

surgery, anterior chamber biometry and control of intraocular lens position stability. ${ }^{18}$

\section{ADVANTAGES OF TECHNOLOGY}

The Pentacam comprehensive eye scanner differs fundamentally from the Orbscan (Bausch and Lomb, Rochester, NY) by the way in which it takes image slices of the cornea. The Orbscan takes vertical image slices that are separated from one another and have no common point. Thus, the Orbscan cannot reregister for any eye movement that occurs while it is capturing the images. Pentacam maintains the central point (the thinnest point) of each meridian. Thus, during the examination, the software can re-register these central points and eliminate the eye movement.

Pentacam can map any structure in the anterior segment that is not opaque. It offers a myriad of clinical applications in a quick and easy to use interface. Another key advantage is that the inter-operator reliability is higher using the automatic release mode, which allows the technicians to use the machine. It measures both the anterior and posterior corneal surfaces, calculating the power of the anterior surface using the difference between the refractive index of air $(n=1)$ and the refractive index for corneal tissue $(n=1.376)$. The power of the posterior surface is calculated using the difference between the refractive index for corneal tissue $(\mathrm{n}=1.376)$ and the refractive index for aqueous humor $(\mathrm{n}=1.336)$. This provides us with a map of the True net corneal power that can differ quite significantly from Placido-based topographic values which use a refractive index of 1.3375 and do not consider posterior surface, especially in patients with previous refractive surgery.

The different zones within pathologic corneal tissues are visible on Scheimpflug images due to their individual light scattering properties. The scattering observed with a slit-lamp or Scheimpflug camera is known as backward scattering and must be distinguished from forward scattering toward the retina.

Corneal elevation, slope, and curvature are three different measurements. Understanding their derivation and 
measurements becomes extremely important when analyzing the cornea for prospective refractive surgery.

Corneal elevation is the measurement of height between two different points at different elevations. Elevation data is a primary data source for the Pentacam.

Slope is the primary data source for Placido-disk- based technology. The slope or the gradient is commonly used to describe the measurement of the steepness, incline, or grade of a straight line from two points with different elevations. A corneal topographer derives slope data from the reflection of concentric rings of light measuring the topography of the cornea.

Slope is the first derivative of elevation. It is then used to calculate either elevation or curvature.

Curvature is the rate of change of the slope. It is the second derivative of elevation and is calculated from slope data from the topography. Thus, looking at the source data, a topographer calculates the curvature and elevation from the slope, whereas the Pentacam uses elevation as its primary source data. This is an important distinction, because a topographer calculates (not measures) elevation data and abnormal or surgically altered corneas may have multiple elevation solutions from these derived calculations. Since the Pentacam's primary data source is elevation, the first and second derivatives (slope and curvature) can be accurately calculated.

\section{METHOD OF IMAGE ACQUISITION}

Pentacam is a rotating Scheimpflug camera that offers a noninvasive way of assessing the anterior chamber of the eye. It takes about 2 seconds to generate an image of the anterior eye. It can acquire 12, 25, 50 images in single scan. The patient is seated with his or her chin on the chinrest and forehead against the forehead strap and asked to fixate straight ahead on the fixation target (blue circular ring). The room lights are switched off for all examinations to get a reflex free image. The operator focuses and aligns real-time image of the patient's eye on the computer monitor, with the machine marking the pupil edge, center and the corneal apex. Arrows displayed on the screen guide the operator to align the instrument in the horizontal, vertical and translatory axes. To reduce operator-dependent variables, the automatic release mode is used wherein it automatically determines when the correct focus and alignment with the corneal apex has been achieved and then captures a scan. The anterior surface of the cornea is calculated with no optical distortion and the tear film has no effect on measurements. Each successive layer, such as the posterior corneal surface and anterior lens surface, is calculated by ray tracing, with the calculation taking into account optical distortion. It has quality check in the form of quality factor (QS) both for image analysis of anterior and posterior corneal surface and values of $>95 \%$ are chosen for analysis.

\section{CLINICAL APPLICATIONS}

\section{Pachymetry}

The Pentacam is the only instrument that measures and analyses the center of the cornea precisely. This offers a significant advantage in accurate corneal topography measurements prior to refractive surgery. For calculating corneal thickness, the camera measures from the top of the epithelium to the anterior surface of the endothelium. Importantly, it does not measure the tear film. Accurate posterior elevation data is a requisite for accurate pachymetry, because pachymetry is simply the difference between the anterior and posterior surfaces. The corneal thickness is displayed as a color image over its entire area from limbus to limbus. The actual thickness can be evaluated at any particular location by clicking on the area or by using the numerical function. Important parameters like thickness in the pupil center, apical corneal thickness and the thinnest location are also provided. The distance and position of the thinnest point relative to the apex of the cornea are also available.

\section{REFRACTIVE SURGERY}

\section{Scheimpflug Imaging in Post-LASIK}

Ciolino and Belin ${ }^{17}$ studied changes in posterior corneal elevation following Excimer treatment in 121 myopic eyes using the Pentacam. Although trend lines suggested a relationship between a thinner corneal and more negative posterior corneal surface in LASIK, photorefractive keratectomy (PRK) trend lines are essentially flat. There was no significant difference between the posterior displacement between LASIK and PRK eyes. No LASIK eyes showed significant forward displacement. They concluded that subclinical incidence of post-LASIK ectasia, previously shown on Orbscan measurement may not be as frequent as reported on Orbscan.

Pentacam is indispensable tool for pre- and postrefractive surgery evaluation of cornea. One of the keys for successful refractive surgery is to be able to identify with fair accuracy the patients with ectasia and forme fruste keratoconus. Corneal thickness spatial profile and corneal volume distribution on the Pentacam have been shown to be useful tool for identifying ectatic conditions. As investigators suggest that changes in posterior corneal curvature are of immense importance in early identification of ectatic conditions, it is important to have an accurate method of studying posterior corneal elevation in postLASIK eyes. These parameters have been shown to have excellent repeatability in post-LASIK corneas on Pentacam. ${ }^{18}$ Pentacam provides maps based on elevation data independent of axis, orientation and positioning unlike systems based on the assumption that all corneas share a common conic section. 


\section{Keratoconus Screening}

Keratoconus screening requires the use of topography systems that measure elevation data as their elemental unit of measurement. Derived elevation (curvature) is not accurate. Pentacam is the only eye screening system that does not rely on Placido-based technology and allows direct measurement of elevation data. The Keratoconus module uses a built in artificial intelligence (neural networks) algorithm for detection of keratoconus based on corneal thickness spatial profile ${ }^{19}$ and quantification; staging it as KK 1, 2, 3 or 4 .

Quisling et al ${ }^{20}$ compared measurements from Orbscan IIz and the Pentacam in eyes with keratoconus. The posterior elevation was significantly different, despite similar radii of curvature. This may be due to a difference in data analysis by the two machines. The Orbscan estimates the central $3 \mathrm{~mm}$ while the Pentacam images the cornea directly. It was suggested that the Orbscan IIz may be more accurate in the periphery but less so in the center, overestimating the posterior height.

Progression of keratoconus can be monitored. Following treatment with newer technologies like corneal collagen crosslinking, (Figs 3A to F) the corneal topography can be followed up to evaluate the changes in the cornea and the stabilization can be verified ${ }^{21}$

\section{Corneal Pathologies}

We have found the Pentacam useful in planning and screening Intacs for keratoconus patients, and in managing post-PKP patients especially in decision making with respect to suture removal.
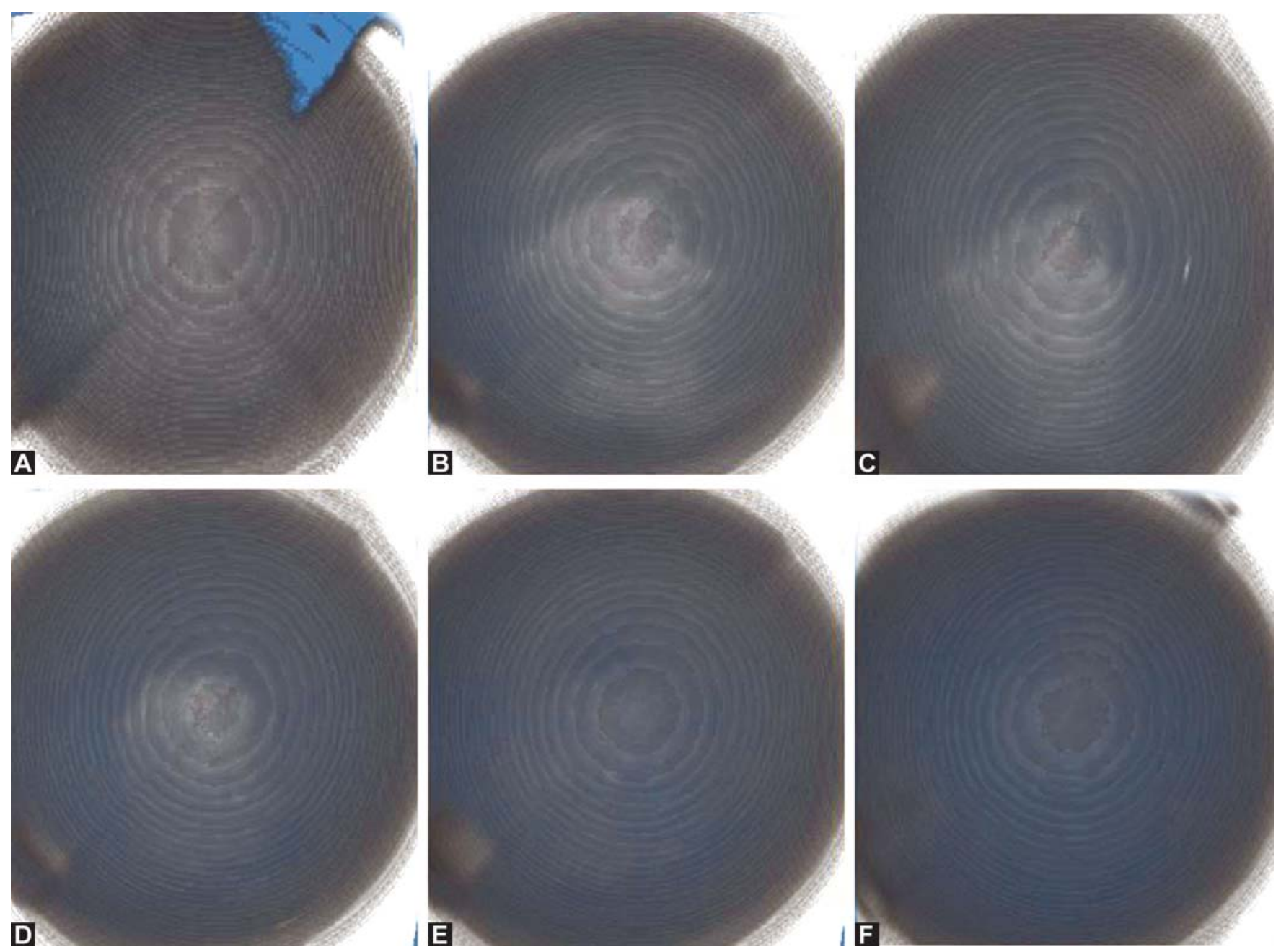

Figs 3A to F: (A) 3D imaging of cornea with keratoconus (preoperative image), (B) One week post C3R image of the same cornea showing corneal haze, (C) One month post C3R image of the same cornea showing corneal haze, (D) Four months post C3R image of the same cornea showing more localized corneal haze (decreasing in size and intensity), (E) Six months spost C3R corneal haze has completely disappeared (F) A clear cornea 2 years post C3R 
It has been an extremely useful to precisely identify subtle corneal opacities and follow the changes over time such as in adenoviral keratoconjunctivits. The response to medical management in such cases can also be assessed.

It helps in monitoring the corneal haze that might occur in patients of surface PRK (Figs 4A to D). High Resolution Pentacam can help in defining the flap-bed interface and hence, can visualize the pathologies occurring at the interface e.g. Interface fluid syndrome that may occur in post-LASIK steroid responders.
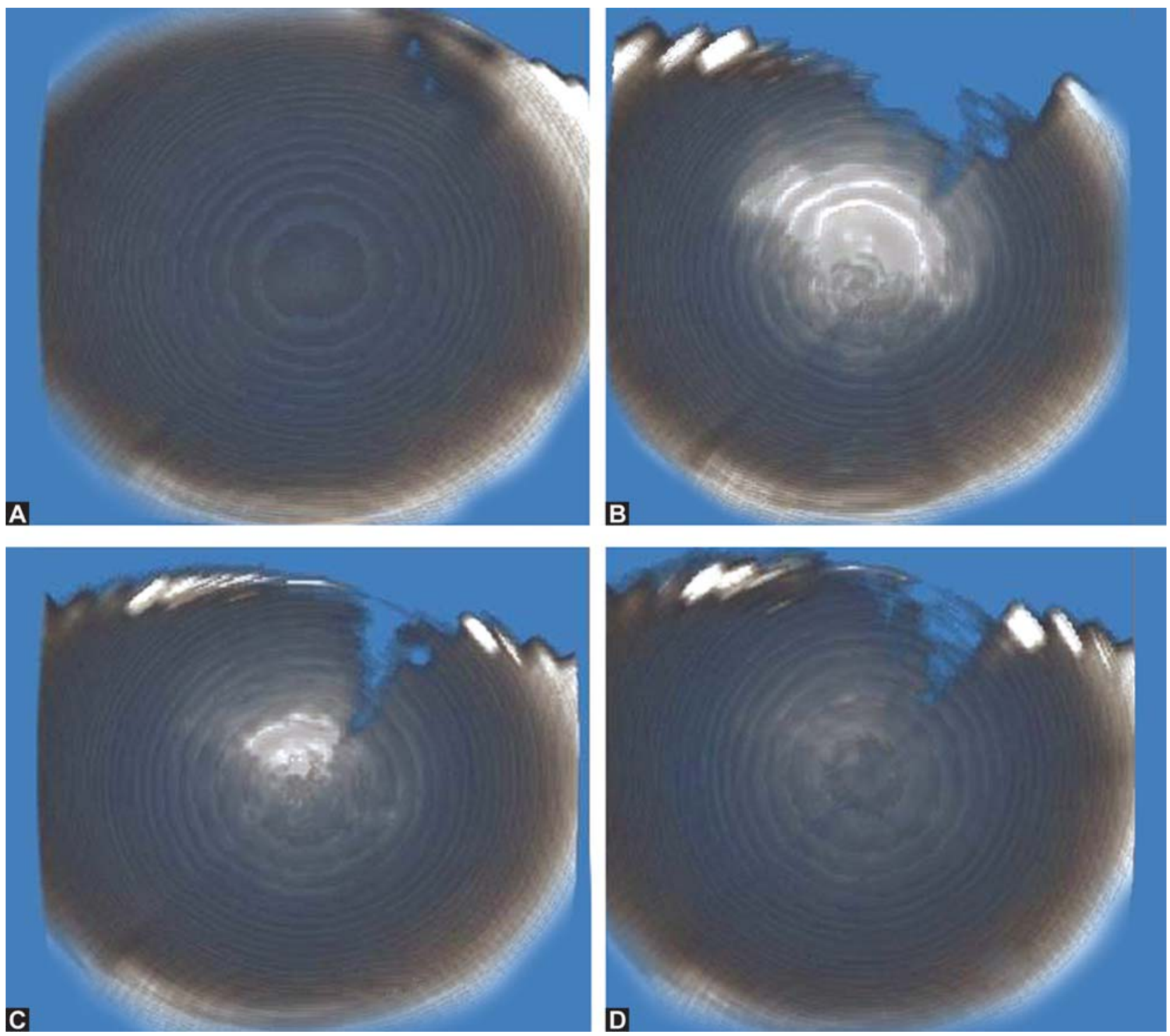

Figs 4A to D: (A) 3D imaging of cornea prealcohol assisted LASEK, (B) One week postalcohol assisted LASEK showing corneal haze secondary to infection, (C) Two months postalcohol assisted LASEK showing persistent corneal haze, (D) Eight months postalcohol assisted LASEK showing more localized corneal haze (decreasing in size as well as intensity)
The 3D chamber analyzer in the software is helpful for surgeons implanting phakic IOL's during the preoperative assessment. Many phakic IOLs, such as toric lenses, require precise implantation in terms of the location of the iris. The more a lens is displaced from the target axis, the greater the induced astigmatism. For example, even $15^{\circ}$ of displacement equals approximately a $50 \%$ reduction in the quality of the refractive effect.

\section{Anterior Chamber}




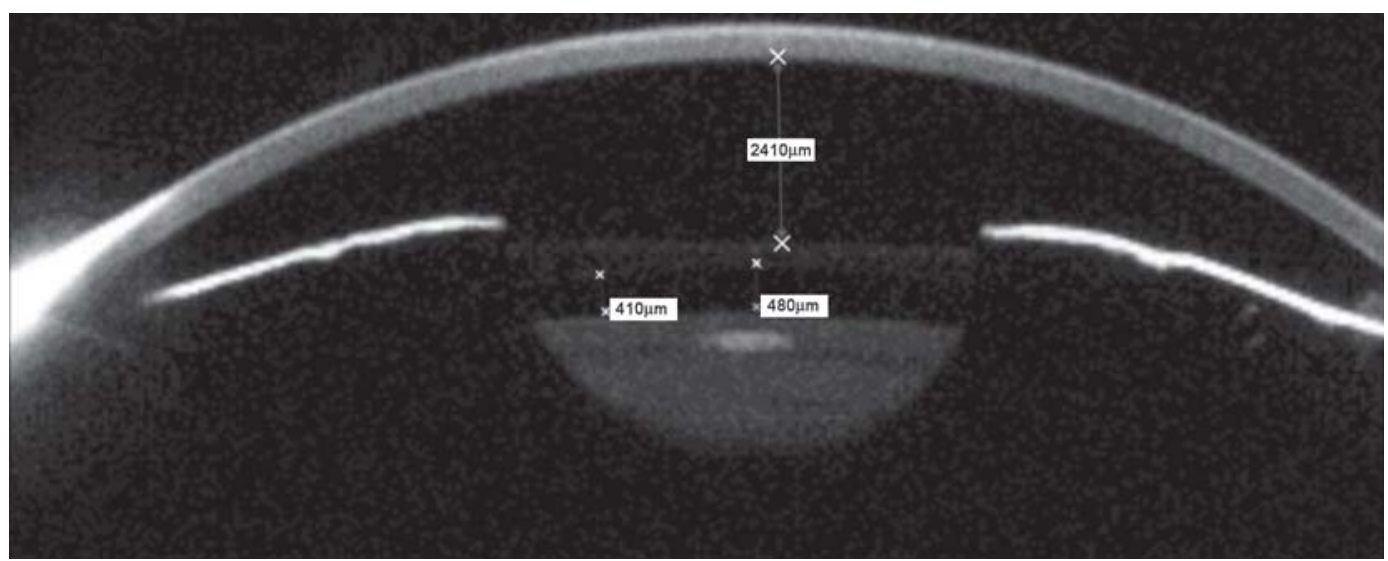

Fig. 5: Implantable contact lens and its position in relation to cornea and anterior lens surface

Pentacam effectively and reliably illustrates the location of implantable contact lens (ICL) positioning in relation to crystalline lens as well as cornea in a repeatable, noncontact quick method (Fig. 5). This allows us the ophthalmologist to follow-up these patients with ICL for monitoring their risk for developing cataract. It also allows for visualization and followup of pre-existing or newly developed anterior subcapsular opacification.

\section{Assessment of Lens Density}

Scheimpflug images allow for an automatic and objective quantification of the lens density. This is available either by means of a built in lens-density module or the Scheimpflug images can be exported to image analysis software. This is useful both in analyzing opacities due to age like nuclear sclerosis and cases of traumatic cataract. ${ }^{22-23}$ The Scheimpflug map of a cataract may show opacities that scatter light and degrade vision. Thus, the physician can show these maps to the patient and document the changes that are occurring to the patient's crystalline lens in his chart.

The Pentacam's densitometry software may help in simplifying cataract grading that provides a reliable, reproducible way of sampling the volume and density of the nuclear cataract and objectively classifying it from grade zero up to a grade 5 other than its objectivity in defining congenital cataracts, traumatic ruptures, etc (Figs 6A to I).

The Pentacam's new densitometry software is not without limitations. A poorly dilated pupil interferes with the device's sampling technology, and so eyes with pseudoexfoliation, trauma, and intraoperative floppy iris syndrome, for example, are problematic. Also, white cataracts block the system's ability to sample the central nucleus. The grading of lens density through Scheimpflug images has been found to correlate well with the LOCS III grading system for cataracts. ${ }^{24}$

Also, comparison studies with the LOCS III system have shown that the Pentacam better predicts the amount of phacoemulsification energy needed to remove various grades of cataract. ${ }^{25}$

It is also excellent for identifying intralenticular foreign bodies ${ }^{26}$ anterior and posterior subcapsular cataracts.

Another interesting application is in diagnosing capsular bag distension syndrome (CBDS.) ${ }^{27}$ Early stages of CBDS are often missed on a slit-lamp examination. It appears to be an excellent tool for demonstrating Elschnig pearls, posterior capsule opacification and late cases of CBDS.

\section{Posterior Capsule Opacification (PCO)}

Pentacam tomograms allow for objective quantification of PCO which is much easier than slit-lamp retroillumination images as they are free of flash reflections. The tomograms provide an excellent representation of the opacified posterior capsule and reflects the changes following Nd:YAG posterior capsulotomy as well. We could convincingly demonstrate its value in visualizing posterior capsule opacification and its quantification using advanced software analysis ${ }^{28}$ (Fig. 7). It gives a more objective evaluation of posterior capsule.

\section{Improved IOL Calculations}

\section{IOL Calculation}

In cases with previous refractive surgery the keratometric readings based only in anterior corneal curvature, such as those obtained with a Placido-based topography or a keratometer, will give an erroneous value, because they are unable to measure the posterior corneal radius. These instruments also assume that the ratio between the posterior and anterior corneal radii is $82 \%$, a relationship that is changed following all types of ablative procedures. In addition, topographers and keratometers are blind to the exact center of the cornea and must extrapolate this information.

The error in central corneal power calculation is the single most common measurement error resulting in the need for an 

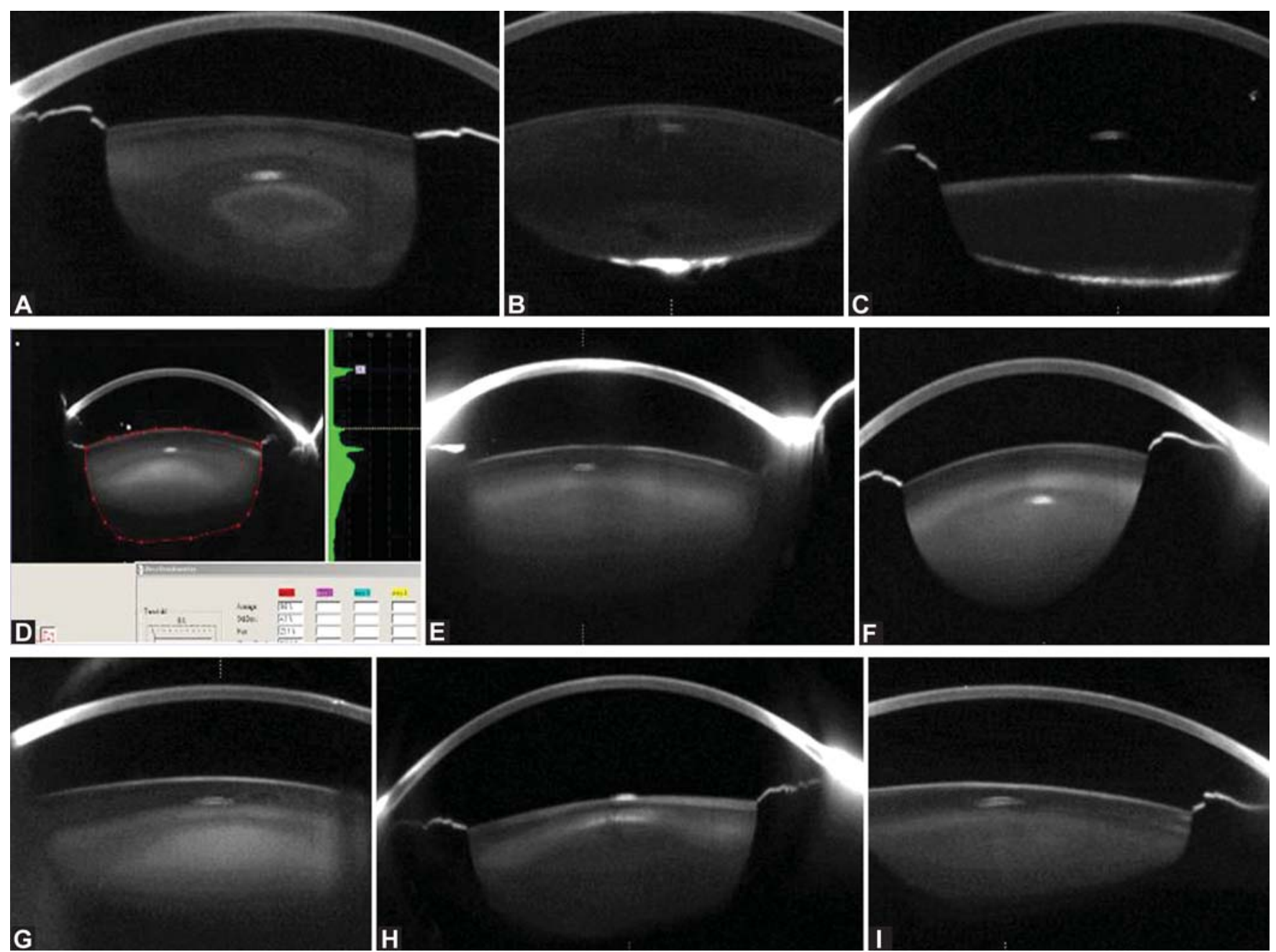

Figs 6A to I: (A) Scheimpflug image on Pentacam of zonular cataract, (B) Scheimpflug image on Pentacam of posterior polar cataract with deficient posterior capsule, (C) Scheimpflug image on Pentacam of Posterior subcapsular cataract, (D) Lens density measurement on Pentacam Scheimpflug imaging, (E to I) Scheimpflug image on Pentacam of varying grades of nuclear cataract

IOL exchange. Where this becomes even more important is for eyes with unusual clinical situations, such as prior ocular trauma, infections or inflammatory events, keratoconus, pellucid marginal degeneration and the various forms of keratorefractive surgery, such as LASIK, PRK and radial keratotomy. The Holladay Report, a module developed by Oculus with Dr. Jack $T$. Holladay gives the physician a realistic measure of the optical power of the cornea at different zones, as well as an estimation of the preoperative simulated K readings. For example, following myopic LASIK, an overestimation of keratometry readings causes an underestimation of IOL power resulting in hyperopic outcomes. Conversely, following hyperopic PRK, an underestimation of the keratometry readings causes an overestimation of the IOL power resulting in myopic outcomes. In the "Holladay Report" incorporated in the Pentacam software the ratio between back and front power of the cornea is calculated for the current examination as well as the "Equivalent K-Readings" of the cornea. These "Equivalent K-Readings" can be implanted into the IOL calculation formulas to get a more precise IOL power calculated for all patients, including those with abnormal corneas.

\section{Glaucoma Screening}

As far as its applications in glaucoma clinic are concerned the parameters like anterior chamber depth, central as well as peripheral; anterior chamber volume; corneal thickness (apical); inbuilt IOP correction formulae and anterior chamber angle have been used in patients with narrow angles.

Following are some of the clinical applications in glaucoma.

1. Effect of pilocarpine on anterior chamber depth and anterior chamber volume in eyes with narrow angle and open angles. Pilocarpine 2\% decreased central anterior chamber depth 

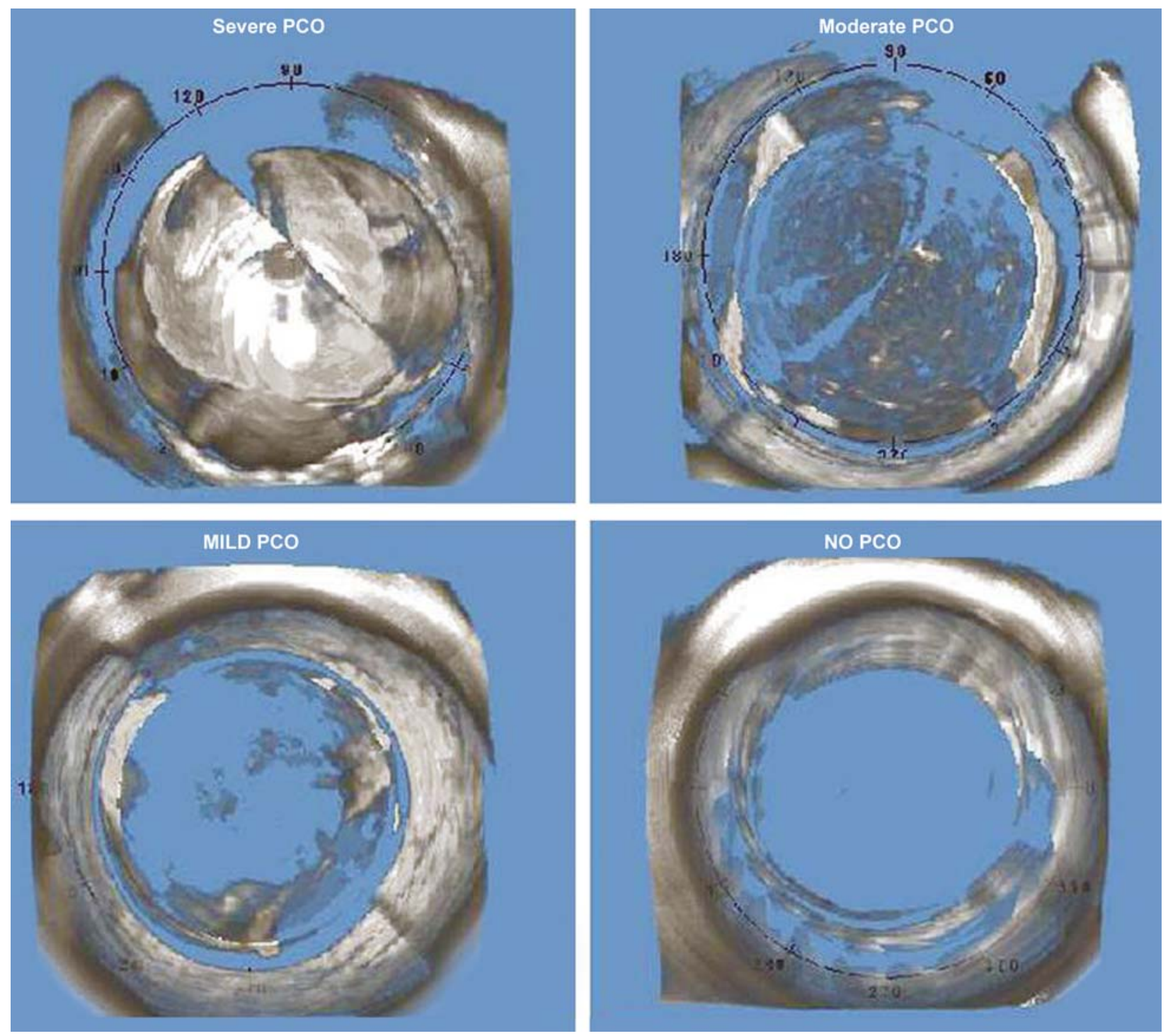

Fig. 7: 3D reconstructive images on Pentacam showing varying grades of posterior capsule opacification

(ACD), chamber volume (ACV) but had angle opening effect by causing relatively less shallowing of Peripheral anterior chamber depth (PACD). Pilocarpine caused shallowing of anterior chamber - central ACD decreased by 97 microns $(p=0.48)$. ACV decreased by $5.7 \mathrm{~mm}^{3}$ which was statistically insignificant $(\mathrm{p}=0.70)$. Maximum shallowing was caused temporally at $4 \mathrm{~mm} .{ }^{29}$

2. Anterior chamber Volume has been found to be a good screening tool for diagnosing eyes with narrow angles (Figs 8A to C). We have found a high sensitivity and specificity for ACV in eyes with narrow angles. The software provides a colored map of the anterior chamber depth-both central and peripheral. With ACV of $110 \mathrm{~mm}^{3}$ as cut off to define narrow angle, the Pentacam had a sensitivity of
88.37\%; Specificity $=90.62 \%$; Positive predictive value $=$ 92.7; Negative predictive value $=85.3$. Any patient having ACV of $<110 \mathrm{~mm}^{3}$ had 9.42 times chance of having a narrow angle on gonioscopy. (Likelihood ratio of having narrow angles $=9.42.)^{30}$

3. The dynamics of the anterior chamber including the ACV can be studied following procedures like laser peripheral iridotomy (PLI). We found that after PLI there was a significant increase in ACV by $28.36 \mathrm{~mm}^{3}$, which was significantly persistent at 1-week and 4-week post PLI. The percentage change in PACD-8 was maximum and the effect of PLI increased with increasing distance from the optical axis (Fig. 9). The central anterior chamber depth did not change significantly immediately after PLI. ${ }^{31}$ 

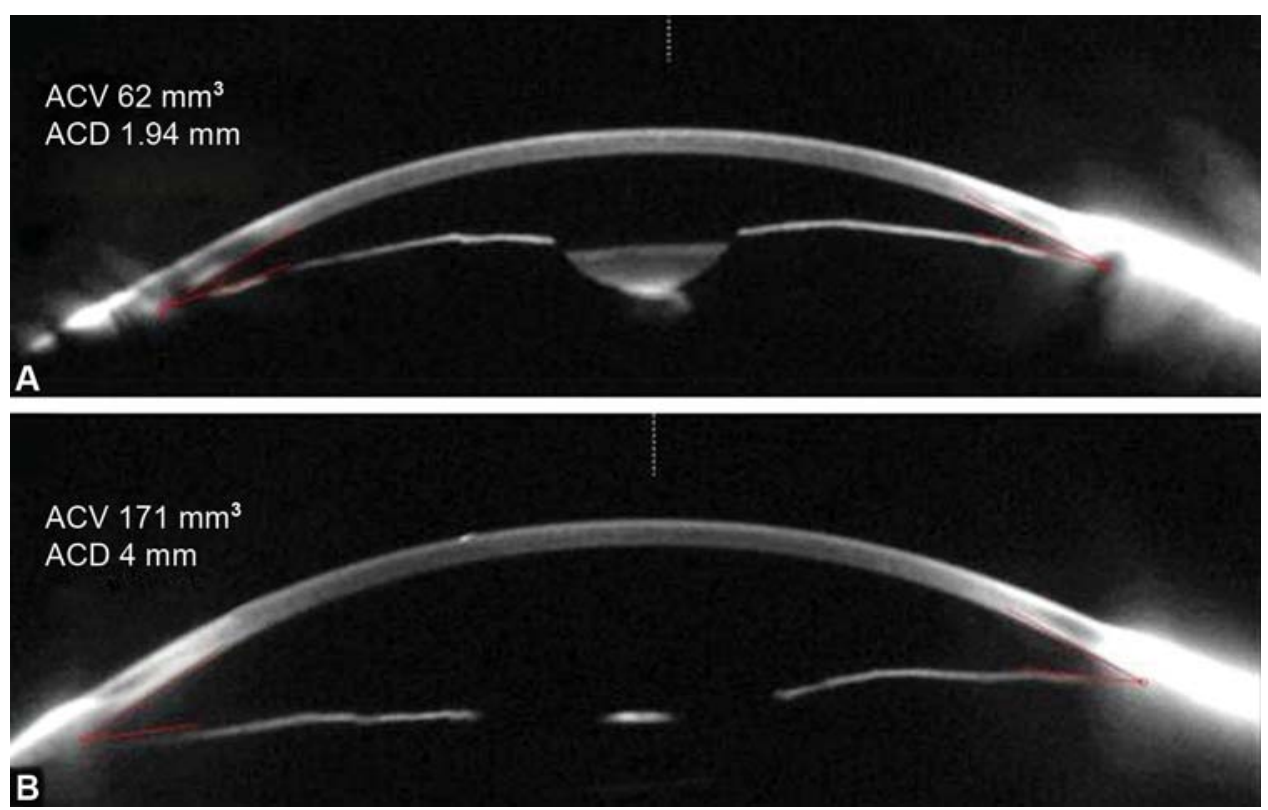

ROC curve

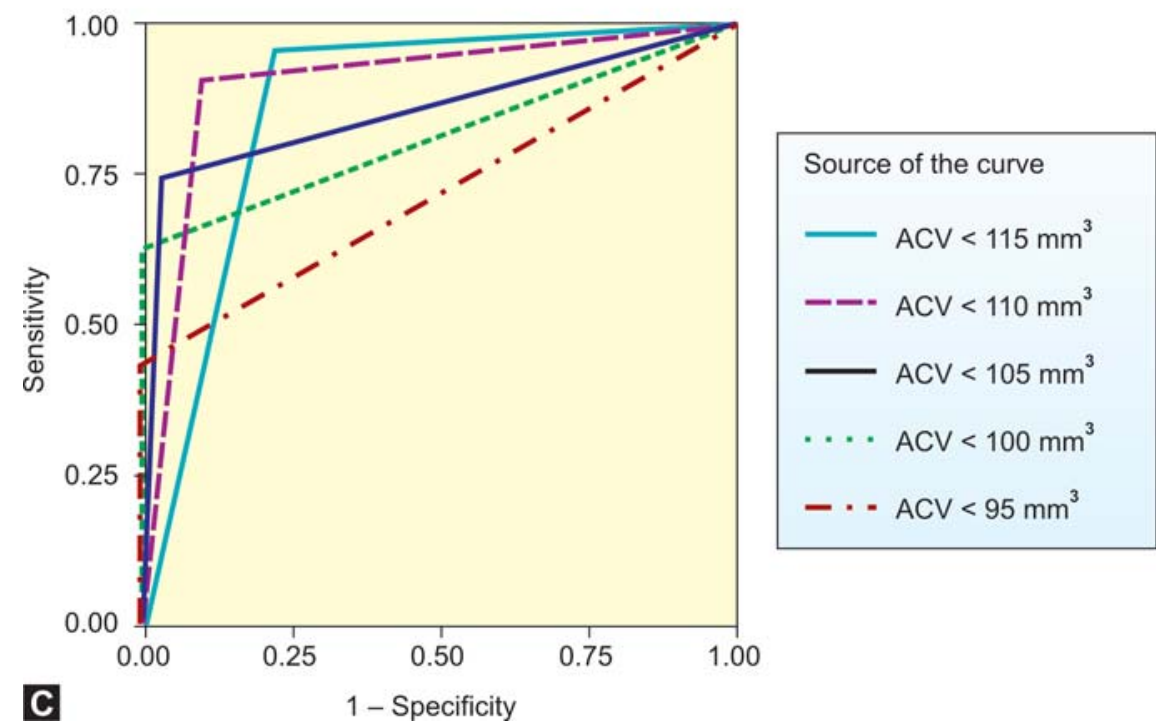

Figs 8A to C: $(A)$ The anterior chamber volume (ACV) and anterior chamber depth (ACD) in a subject with narrow angles on gonioscopy (B) The anterior chamber volume (ACV) and anterior chamber depth (ACD) in a subject with open angles on gonioscopy, (C) The area under $\mathrm{ROC}$ for various ACV values for diagnosis of narrow angle on Pentacam

Oka et $\mathrm{al}^{32}$ reported that the ACV for the narrow angle group (74.5+/-21.1 microl) was significantly smaller than for the other groups (post PLI group: $96.4+/-21.4$ microl; open angle: 144.2 $+/-31.6$ microl, $\mathrm{p}<0.001$ ). The most significant association was detected between ACV and the peripheral AC depth. Only two parameters, ACV and peripheral AC depth, increased significantly after PLI $(\mathrm{p}<0.001)$ and concluded that the measurement of the AC volume and the peripheral AC depth using Pentacam is useful for evaluating the anterior ocular segment topography in narrow angle eyes.
Various IOP correction formulae are incorporated in the Pentacam software based on the central corneal thickness (Fig. 10).

However it is limited in its evaluation of the anterior chamber angle ${ }^{33}$ as compared to the anterior segment OCT. This is due to the reflectance from the scleral surface and hence, inability to visualize the sclera spur.

Direct angle visualization of the scleral spur, ciliary body, and ciliary sulcus is possible only with the anterior segment OCT and UBM; not the Pentacam. 


\section{OCULUS - PENTACAM}

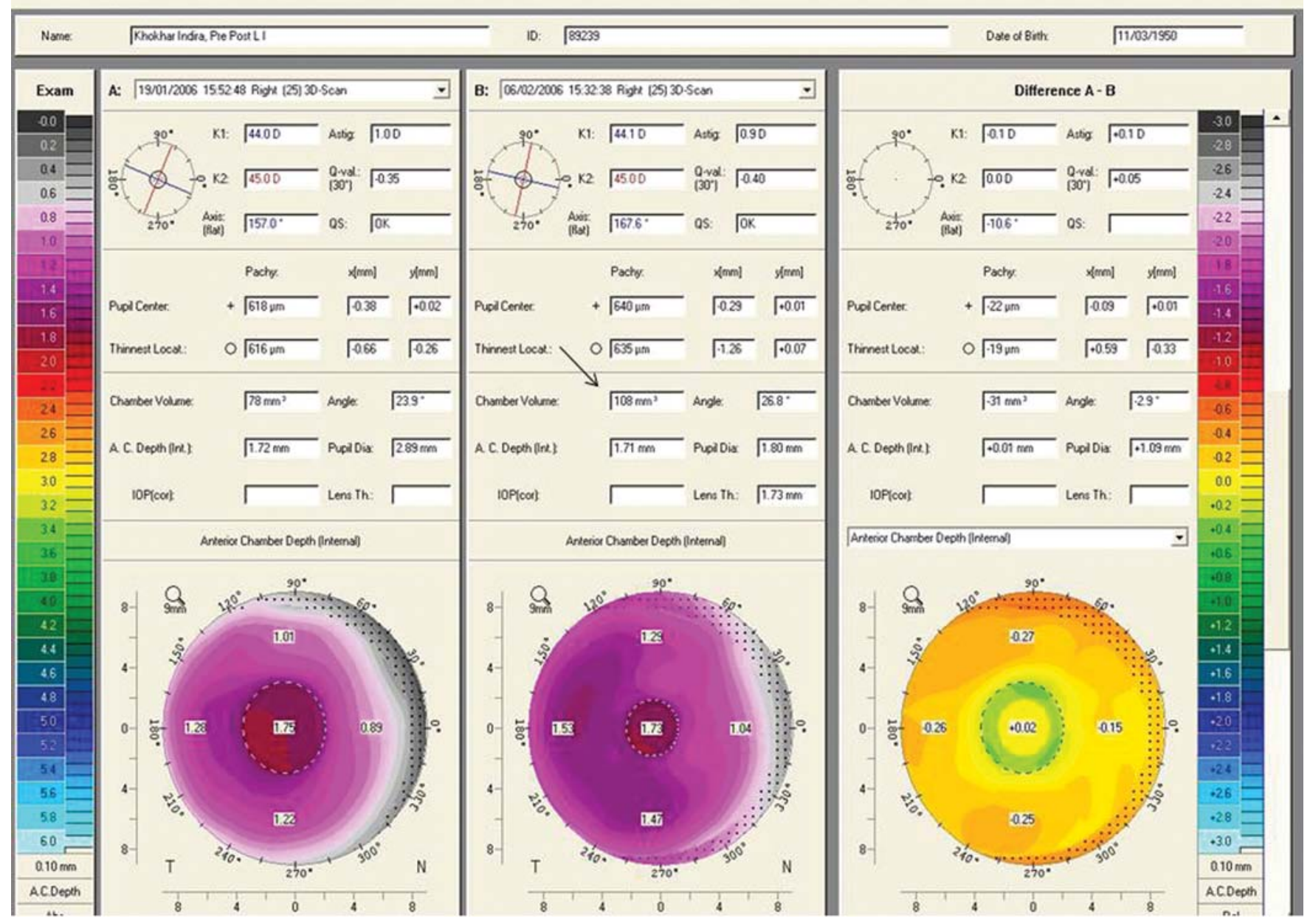

Fig. 9: Difference map on Pentacam highlighting the increase in anterior chamber volume (arrow)

following peripheral laser iridotomy and no change in central anterior chamber depth

\section{New High Resolution (HR) Pentacam}

The new High Resolution Pentacam captures 100 images in less than 2 seconds using a higher resolution 1.45 Mega-Pixel Camera. It provides sharper Scheimpflug pictures allowing for more detailed IOL and phakic IOL implant imaging. Additionally it allows more precise imaging of the corneal layers, which can be used to evaluate the flap parameters in refractive surgery, similar to the anterior segment OCT.

\section{CONCLUSION}

In conclusion, Pentacam's ability to measure and visualize the anterior segment is currently unsurpassed. It is an extremely versatile tool for a clinician and also provides immense scope for research. Pentacam is of great utility in daily clinical practice and provides a wealth of information. It's easy to use nature; reproducibility and rapid scanning ability make it an indispensable tool for the modern anterior segment ophthalmologist. It can be effectively utilized in glaucoma screening using its objective anterior chamber volume (ACV) parameter.

\section{REFERENCES}

1. Scheimpflug T. Der photoperspektrograph und seine anwendung. Photogr Korr 1906;43:516-31.

2. Mayer H. Theodor Scheimpflug, his personality and lifework. Ophthalmic Res (Suppl. 1) 1994;26:3-9.

3. Scheimpflug T. Verwendung des Skioptikons zur Herstellung von Karten und Plänen aus Photographien, 69. Braunschweig: Vers. der Naturforscher und Ärzte, 1897.

4. Drews C. Depth of field in slit image photography. An optical solution using the Scheimpflug principle. Ophthalmologica 1964;148:143-50.

5. Niesel P. Spaltlampenphotographie der Linse für Messzwecke. Ophthalmologica 1966;152:387-95. 


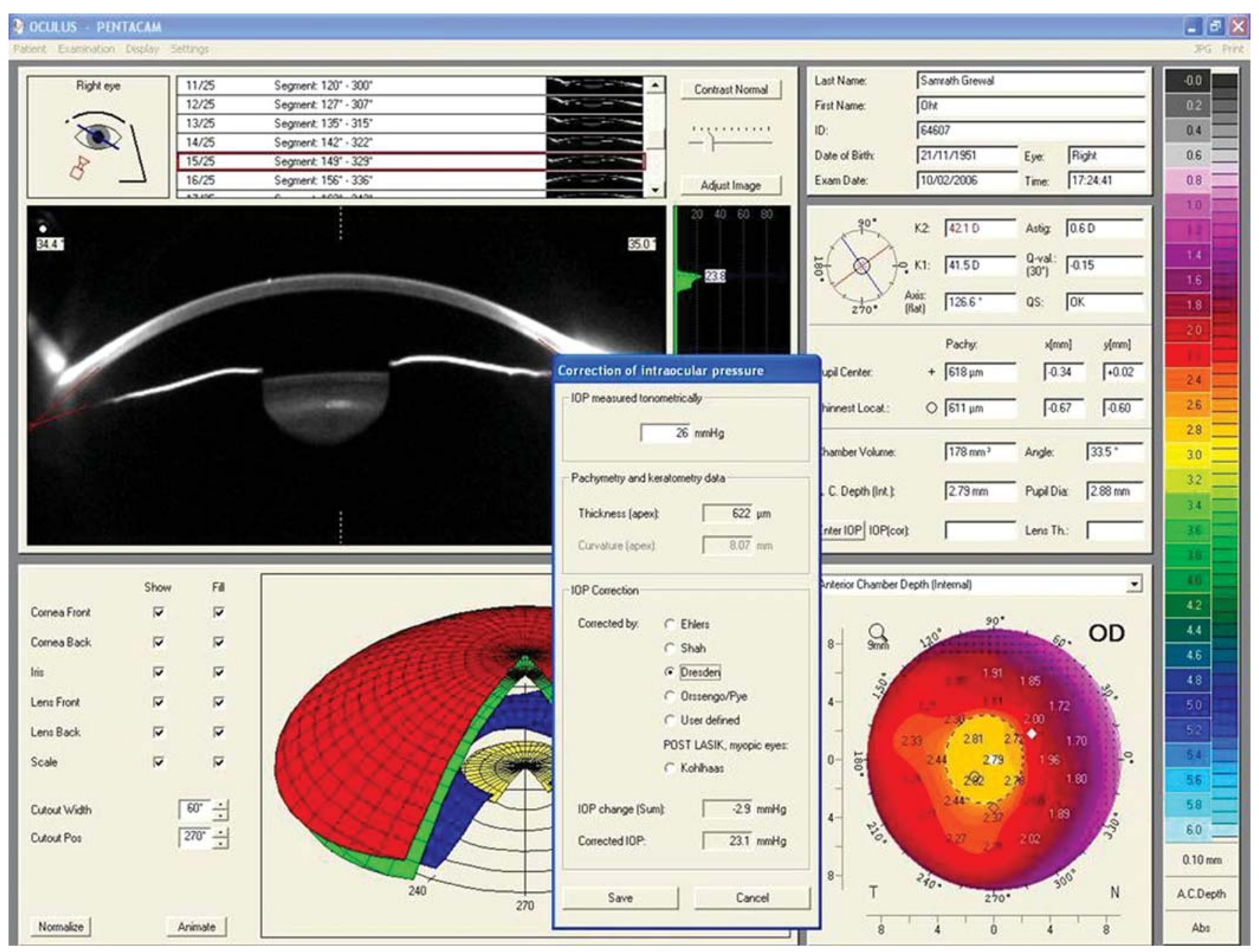

Fig. 10: Various Intraocular pressure correction formulae are incorporated in the Pentacam software based on the central corneal thickness

6. Brown NAP. Slit image photography. Trans Ophthalmol Soc UK 1969;89:397-408.

7. Brown N. Quantitative slit-image photography of the lens. Trans Ophthalmol Soc UK 1972;92:303-17.

8. Brown N. Lens changes with age and cataract: slit image photography. In Ciba Foundation Symp. The Human Lens in Relation to Cataract. Amsterdam: Exerpta Medica/North Holland, Elsevier 1973;19:65-78.

9. Dragomirescu V, Hockwin O, Koch H-R, Sasaki K. Development of anew equipment for rotating slit image photography according to Scheimpflug's principle. Interdiscip Top Gerontol 1978;13:118-30.

10. Hockwin O, Dragomirescu V, Laser H, Wegener A. Scheimpflug photography of the anterior eye segment. Principle, instrumentation and application to clinical and experimental ophthalmology. J Toxicol Cutaneous Ocul Toxicol 1987;6:251-71.

11. Sparrow JM, Brown NA, Shun-Shin GA, Bron AJ. The Oxford modular cataract image analysis system. Eye 1990;4:638-48.
12. Sasaki K, Sakamoto Y, Shibata T, Emori Y. The multipurpose camera: A new anterior eye segment analysis system. Ophthalmic Res 1990;22(Suppl. 1):3-8.

13. Rabsilber TM, Khoramnia R, Auffahrt GU. Anterior chamber measurements using Pentacam rotating Scheimpflug camera. J Cataract Refract Surg 2006;32:456-59.

14. Shankar H, Taranath D, Santhirathelagan CT, Pesudovs K. Anterior segment biometry with Pentacam: Comprehensive assessment of repeatability of automated measurements. J Cataract Refract Surg 2008;34:103-13.

15. Dubbelman M, Van der Heijde RGL. The shape of the aging human lens: Curvature, equivalent refractive index and the lens paradoxon. Vision Res 2001;41:1867-77.

16. Dubbelman M, Weeber HA, Van der Heijde RGL, Völker-Dieben HJ. Radius and asphericity of the posterior corneal surface determined by corrected Scheimpflug photography. Acta Ophthal Scand 2002;80:379-83. 
17. Ciolino JB, Belin MW. Changes in the posterior cornea after laser in situ keratomileusis and photorefractive keratectomy. J Cataract Refract Surg 2006;32:1426-31.

18. Jain R, Grewal D, Grewal SP. Repeatability of corneal parameters with Pentacam after laser in situ keratomileusis. Indian J Ophthalmol 2007; 55(5):341-47.

19. Quisling S, Sjoberg S, Zimmerman B, et al. Comparison of Pentacam and Orbscan IIx on posterior curvature topography measurements in keratoconus eyes. Ophthalmology 2006;113:1629-32.

20. Ambrosio R, Alonso RS, Luz A, Coca Velarde LG. Cornealthickness spatial profile and corneal-volume distribution: Tomographic indices to detect keratoconus. J Cataract Refract Surg 2006;32:1851-59.

21. Grewal D, Brar GS, Jain R, et al. Corneal collagen crosslinking using riboflavin and ultraviolet-A light for keratoconus: Oneyear analysis using Scheimpflug imaging. J Cataract Refract Surg Mar 2009;35(3):425-32.

22. Grewal DS, Jain R, Brar GS, Grewal SP. Posterior capsule rupture following closed globe injury: Scheimpflug imaging, pathogenesis, and management. Eur J Ophthalmol 2008 May-Jun;18(3):45355.

23. Grewal DS, Jain R, Brar GS, Grewal SP. Unilateral electric cataract: Scheimpflug imaging and review of the literature. J Cataract Refract Surg Jun 2007;33(6):1116-19.

24. Grewal D, Brar GS, Jain R, Grewal SPS. Corelation of lens density on Pentacam images with Lens Opacities Classification system III ad visual performance. Presented as paper at: The Annual meeting of American Academy of Ophthalmology, November 10-13, 2007; New Orleans, Lousiana.

25. Nixon DR. Redefining cataract grading to enhance transferability and reproducibility for future phaco programming. Paper presented at: The Annual Meeting of the ASCRS; April 30, 2007; San Diego, CA.

26. Grewal SPS, Jain R, Gupta R, Grewal D. Role of Scheimpflug imaging in traumatic intralenticular foreign body. Am J Ophthalmol Oct 2006;142(4):675-76.

27. Jain R, Grewal D, Gupta R, Grewal SPS. Scheimpflug imaging in late Capsular Bag Distention syndrome after phacoemulsification. Am J Ophthalmol Dec 2006;142(6):1083-85.

“Imagination is more important than knowledge."
28. Grewal D, Jain R, Brar GS, Grewal SPS. Pentacam tomograms: a novel method for quantification of posterior capsule opacification. Invest Ophthalmol Vis Sci 2008;49:2004-08.

29. Jain R, Grewal D, Grewal SPS. Pentacam evaluation of changes in Anterior Chamber Depth and Volume in cases with Primary Angle Closure after instillation of Pilocarpine. Presented at: The XXIV Annual Congress of ESCRS; September 2006;9-13, London, United Kingdom.

30. Jain R, Grewal D, Grewal SPS. Predictive value of Anterior Chamber Volume on Scheimpflug Imaging in Eyes with Narrow Angle. Presented at: The Asia ARVO meet; March 2-5, 2007; Singapore and World Glaucoma Congress; July 18-21, 2007; Singapore.

31. Jain R, Grewal D, Grewal SPS. Changes in anterior chamber depth and Volume following laser peripheral laser iridotomy in eyes with Primary Angle closure using Pentacam. Presented at: The Asia ARVO meet; March 2-5, 2007; Singapore and World Glaucoma Congress; July 18-21, 2007; Singapore.

32. Oka N, Otori Y, Okada M, et al.[My paper] Clinical study of anterior ocular segment topography in angle-closure glaucoma using the three-dimensional anterior segment analyzer Pentacam. Nippon Ganka Gakkai Zasshi. 2006 May;110(5):398-403.

33. Yi J, Lee H, Hong S, et al. Anterior Chamber Measurements by Pentacam and AS-OCT in Eyes With Normal Open Angles. Korean J Ophthalmol 2008;22:242-45.

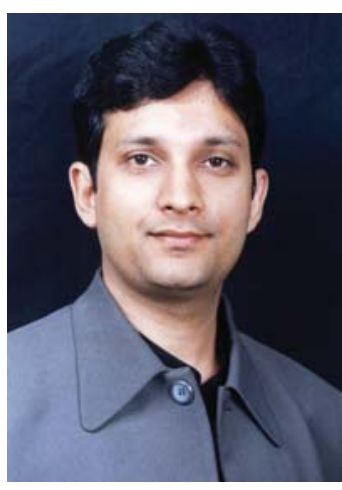

Rajeev Jain (rajeevjain74@gmail.com) 